NASA-TM-112764

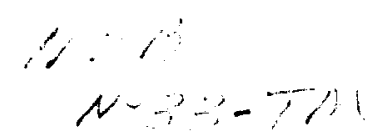

\title{
AUTOMATION OF DATA ANALYSIS PROGRAMS USED IN THE CRYOGENIC CHARACTERIZATION OF SUPERCONDUCTING MICROWAVE RESONATORS
}

\author{
A. S. Creason ${ }^{*}$ and F. A. Miranda \\ National Aeronautics and Space Administration \\ Lewis Research Center \\ Cleveland, OH 44135, USA
}

\begin{abstract}
Knowledge of the microwave properties at cryogenic temperatures of components fabricated using High-Temperature-Superconductors (HTS) is useful in the design of HTSbased microwave circuits. Therefore, fast and reliable characterization techniques have been developed to study the aforementioned properties. In this paper, we discuss computer analysis techniques employed in the cryogenic characterization of HTS-based resonators. The revised data analysis process requires minimal user input, and organizes the data in a form that is easily accessible by the user for further examination. These programs retrieve data generated during the cryogenic characterization at microwave frequencies of HTS based resonators and use it to calculate parameters such as the loaded and unloaded quality factors $\left(Q\right.$ and $Q_{0}$, respectively), the resonant frequency $\left(\mathrm{f}_{0}\right)$, and the coupling coefficient $(\kappa)$, which are important quantities in the evaluation of HTS resonators. While the data are also stored for further use, the programs allow the user to obtain a graphical representation of any of the measured parameters as a function of temperature soon after the completion of the cryogenic measurement cycle. Although these programs were developed to study planar HTS-based resonators operating in the reflection mode, they could also be used in the cryogenic characterization of two ports (i.e., reflection/transmission) resonators.
\end{abstract}

\section{INTRODUCTION}

The discovery of High-Temperature-Superconductors (HTS) in 1986 has resulted in passive planar microwave devices such as resonators and filters with higher quality factors

- NASA/OAI summer intern, Ohio Northern University, Ada, OH 45810, USA.

Advances in Cryogenic Engineering, Vol. 41 

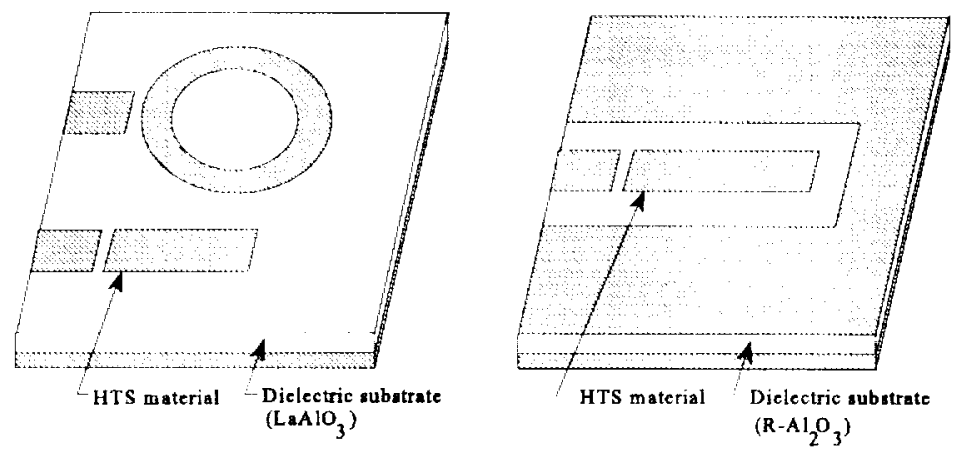

Figure I. YBCO microstrip linear, ring, and conductor-backed coplanar waveguide resonators deposited on $1 \times 1 \mathrm{~cm}, 508 \mu \mathrm{m}(20 \mathrm{mil})$ thick, dielectric substrates

and lower insertion loss than those fabricated using normal conductors ${ }^{1-3}$. Significant attention has been given to the processing and characterization of HTS-based planar microwave components as efforts continue towards the inclusion of HTS-based circuits in working systems for space- and ground-based communications. Therefore, fast and accurate characterization and more advanced data analysis capabilities become extremely important in this endeavor. This paper deals with the data analysis programs used in our laboratory in the study of HTS-based microwave resonators. Extensive modifications of preexisting programs (written in-house for data analysis purposes) were performed in order to expedite the characterization of these resonators without sacrificing accuracy. The structure of these programs will be described. and data taken using $\mathrm{YBia}_{2} \mathrm{Cu}_{3} \mathrm{O}_{7-\delta}$ (YBCO) resonators on lanthanum aluminate $\left.(\mathrm{LaAlO})_{3}\right)$ and $\mathrm{R}$-plane sapphire $\left(\mathrm{R}-\mathrm{Al}_{2} \mathrm{O}_{3}\right)$ substrates will be presented.

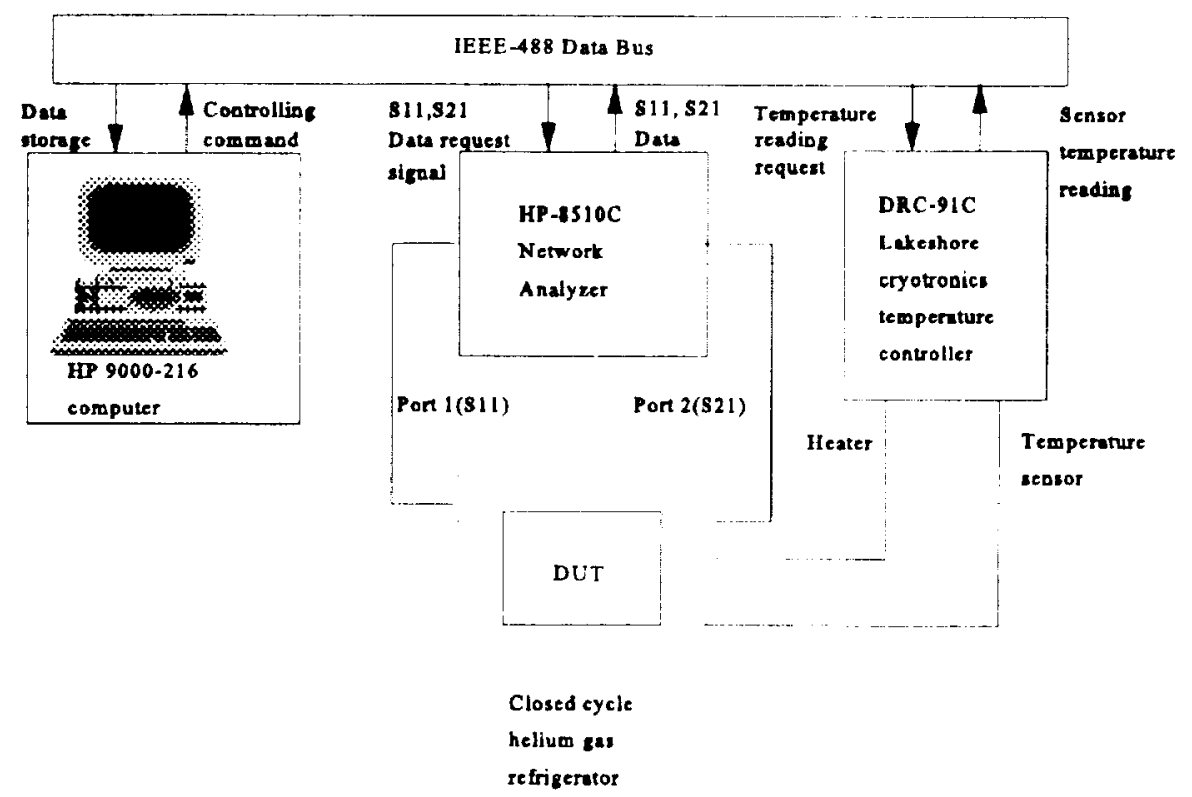

Figure 2. Experimental setup used in cryogenic testing of HTS-based resonators. 


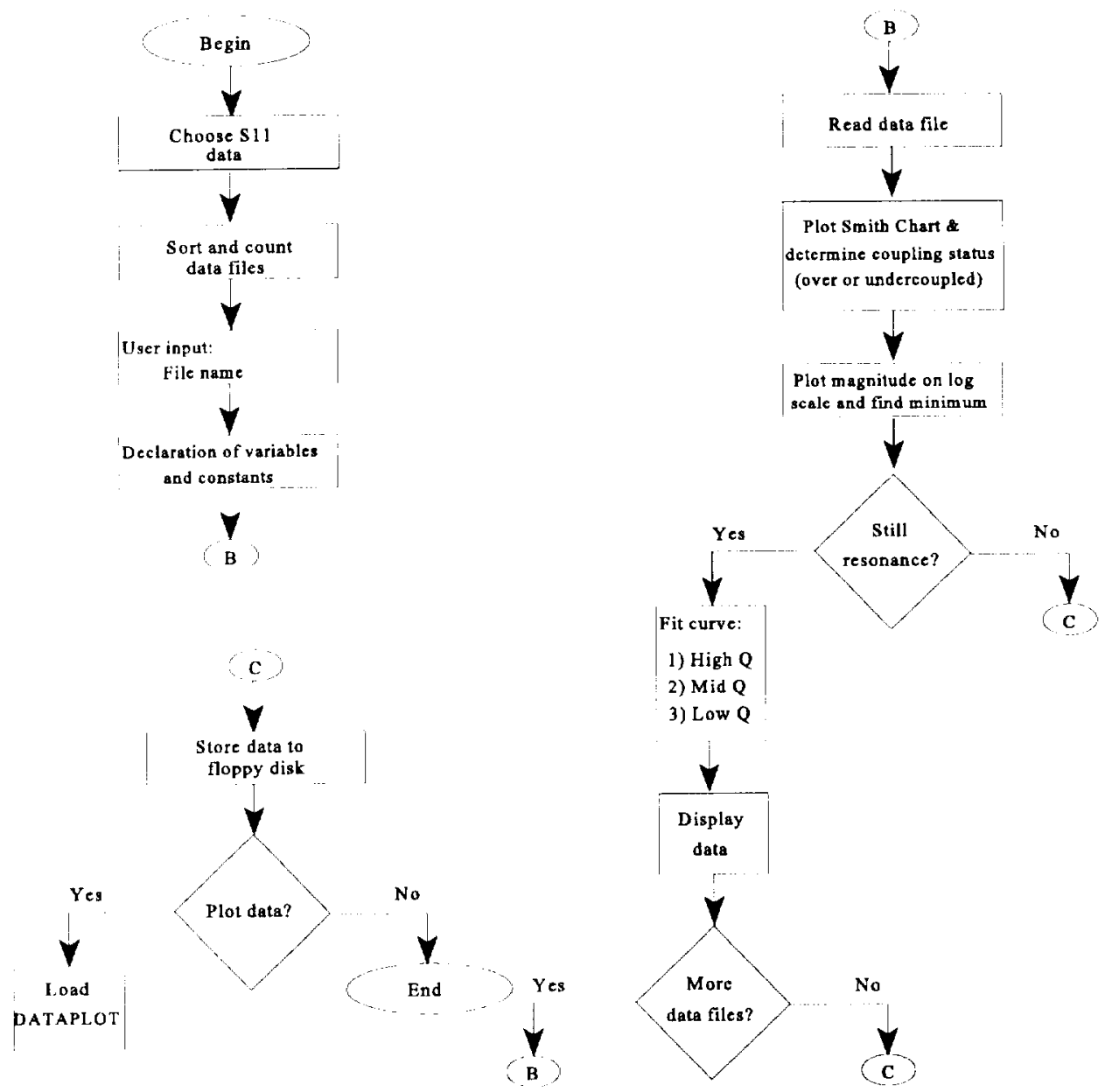

Figure 3. Flowchart for data analysis program 'QANALYSIS'.

\section{EXPERIMENTAL}

Microstrip linear and ring resonators, as well as conductor-backed coplanar waveguide (CBCPW) resonators were fabricated on $\mathrm{YBCO}$ thin films deposited by laser ablation on $\mathrm{LaAlO})_{3}$ substrates and on $\mathrm{R}-\mathrm{Al}_{2} \mathrm{O}_{3}$ substrates (with $\mathrm{CeO}_{2}$ buffer layers), respectively, using standard photolithography and chemical etching techniques. A schematic of these resonators is shown in Figure 1. These resonators were tested by measuring the reflection scattering parameter $\left(S_{11}\right)$, using the experimental configuration shown in Fig $2{ }^{4}$. Briefly, the setup consists of an HP-8510C network analyzer, an HP 9000-216 computer, and a DRC-91C Lake Shore cryotronics temperature controller all coupled through the use of an IEEE-488 data bus, along with a closed-cycle-helium-gas refrigerator containing the device under test (DUT), which allows for cryogenic measurements in the temperature range from $300 \mathrm{~K}$ to $20 \mathrm{~K}$. The data measured through the use of this system were then used by the data analysis programs to determine the loaded and unloaded quality factors $\left(Q\right.$ and $Q_{n}$, respectively), the resonant frequency $\left(f_{i}\right)$, and the coupling coefficient $(\kappa)$ of the resonator.

Figure 3 shows a flowchart for the data analysis program we call 'QANALYSIS', after modification and automation. When automating this program, the first step was to have the 
computer choose the files to be analyzed from the disk. In this way, the user would not have to be familiar with the format used in naming the files in order to choose those which are important in analyzing the data. Data files are named in the following way. The first four characters are always 'DATA'. The next 2 or 3 characters are numerals which show the temperature at which the data was taken. The next character is either a ' $W$ ' or a ' $C$ ', showing whether the data were taken during the warming or cooling cycle. The final two characters are either ' 11 ' or ' 21 ', indicating whether the data are $S_{11}$ (reflection) or $S_{21}$ (transmission). Thus, 'QANALYSIS' can be used for the analysis of one port (reflection mode) as well as two ports (reflection/transmission modes) resonators. Since in this paper we are dealing with one port HTS-based resonators. 'QANALYSIS' catalogs the disk looking for files with the first four characters 'DATA' and the last two '11'. It counts all of the files which fit that requirement, and sets up a counting loop which will analyze only those particular files. When all of the appropriate files have been analyzed, the program ends. The program may terminate execution before all of these files have been analyzed, if certain conditions exist in the data, as will be described later in this paper.

After selecting the data files corresponding to the particular temperatures of interest, the data for the first file in the array is displayed as a Smith Chart plot on the computer's CRT screen. The Smith Chart facilitates the graphical solution of transmission line problems and consists of a plot of the normalized impedance. given by

$$
Z / Z_{0}=r+j x
$$

with the magnitude and phase of a generalized complex reflection coefticient in a unity circle. The chart is useful for the analysis of lossless and lossy lines. It is used in this case to determine the coupling status of the resonator (i.e., overcoupled $(\kappa>1)$, undercoupled $(\kappa<1)$, or critically coupled $(\kappa=1)$ ) where $\kappa$, the coupling coefficient, is defined as

$$
k=\left(l \pm\left|\Gamma_{\mathrm{r}}\right|\right) /\left(1 \mp\left|\Gamma_{\mathrm{r}}\right|\right)
$$

with the sign depending on the coupling status of the circuit. $\Gamma_{r}$ is the reflection coefficient of the circuit at resonance". For $\kappa=1 . \Gamma_{\mathrm{r}}=0$ ) indicating that the resonator is perfectly matched to the source. 'QANALYSIS' calculates the radius of the circle corresponding to the experimental data, then compares that value with that of the circle on the Smith Chart corresponding to critical coupling, which has a radius of 0.5 . If the radius of the plot of the experimental data is less than 0.5 , the circuit is undercoupled, and if it is over 0.5 , the circuit is overcoupled. Next, the program plots the magnitude of the data on a logarithmic scale as a function of frequency. Depending on the quality of the resonator, this plot should show a minimum at or very near the resonant frequency for which the resonator was designed. 'QANALYSIS' then goes on to determine $f_{0}$. This step is implemented such that the correct resonance is selected: i.e., after finding the frequency at which a minimum point occurs, the program searches the entire frequency band under study for any other resonance. This accounts for cases in which a split resonance may occur, as for example, when testing microstrip ring resonators, where secondary or spurious resonances can appear due to variations in thickness, width, etc., of the line ${ }^{7}$. These secondary resonances could be larger than the primary resonance when the circuit is overcoupled. Note that with minor modifications, this searching capability could be applied in the analysis of multipole resonant circuits. The program also verifies that resonance still exits in the data; if the minimum point is not more than $3 \mathrm{~dB}$ below the value of the data where it is flat (i.e., at a frequency far from $f_{0}$ ), then the program indicates that a resonance no longer exists and the analysis is completed.

After the determination of $f_{0}$ is complete, it is then used to calculate the loaded quality factor of the resonator, since 


\section{Q ANALYSIS FOR FILE:DATAT7NII 76.7 KELUIH \\ RESCOANT FREQUENCY IS: \\ UALOADED ' $Q$ ' IS \\ $7.966296196766 \mathrm{~Hz}_{2}$ \\ LOADED ' $Q$ ' IS \\ 1460.35560716 \\ COUPLINE COEFFICIENT IS: 2.29360265636 \\ COUPLING LOSS 1S; $\quad .0987639596878$ \\ FIT: HIGH \\ HALF POUER SPAN IS: $.0538133883643 \quad .0177219568385$}

Figure 4: Sample printout of data from 'QANALYSIS'.

$$
\mathrm{Q}=\left(\mathrm{f}_{0} / \Delta \mathrm{f}\right)
$$

where $\Delta f^{\circ}$ is the half power frequency span.

The final step in analyzing each data file is to fit a curve to the resonance. This assumes that the graph of the resonance resembles a parabola, and uses a second degree polynomial for the curve fitting. Depending on the shape of the curve, e.g. sharp, moderate, or shallow, the program will classify the fit as either High Q. Mid Q, or Low Q

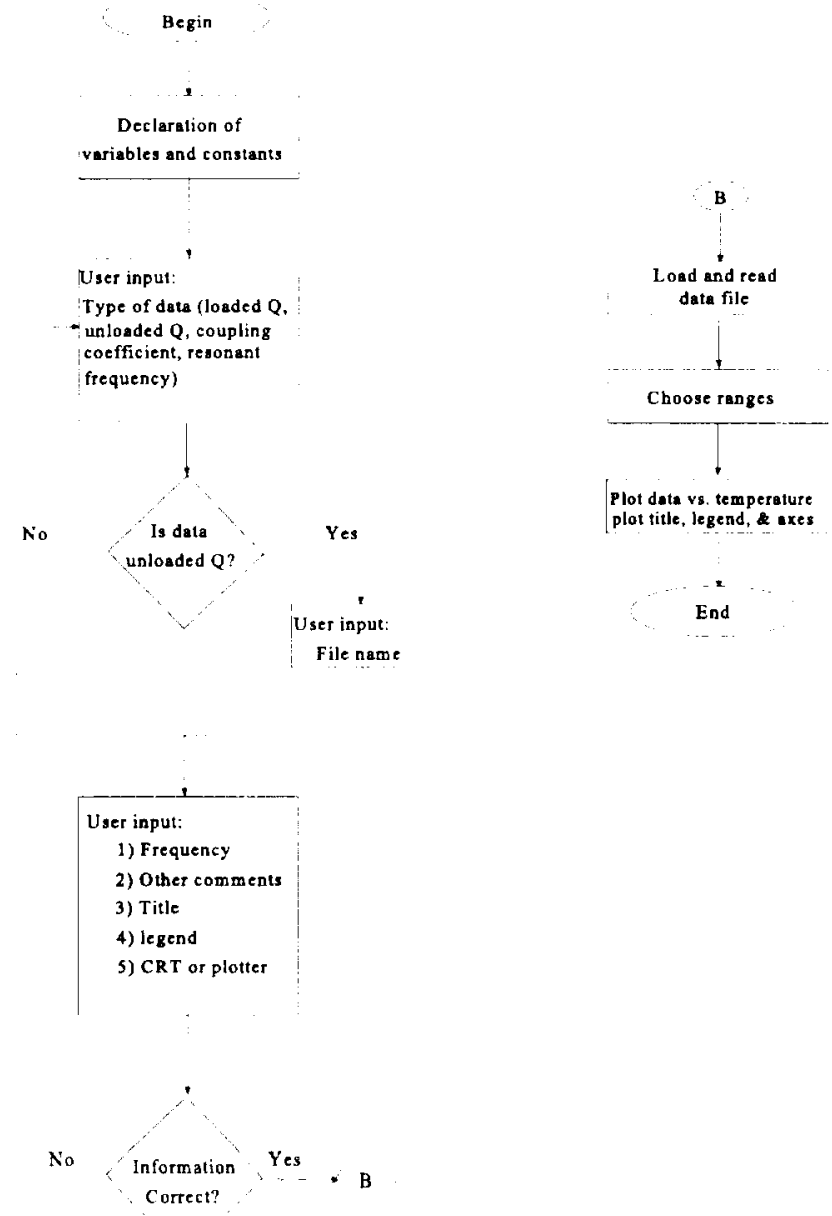

Figure 5. Flowchart for ploting program 'DATAPLOT'. 
respectively, which will be used to aid in the determination of the unloaded quality factor. In calculating $\mathrm{Q}_{3}$, the coupling effects must always be considered. regardless of the coupling state of the resonator. The coupling loss $(\sigma)$, which modifies the location of the half power frequency points. can be found by

$$
\sigma=\left(1-\left|\Gamma_{\infty}\right|\right) /\left(1+\left|\Gamma_{\infty}\right|\right)
$$

where $\Gamma_{\infty}$ is the reflection coefficient of the circuit far from $f_{0}$. In this program, $Q_{0}$ is calculated in a similar manner to $Q$, except that for the calculation of $Q_{0}$, the half power frequency span is that obtained after correcting for coupling losses in the circuit. The program also calculates several other parameters, then prints at list which includes the file's name, the temperature, $Q, Q_{0}, f_{0}, \kappa, \sigma$, the data fit type, and the half power span $(3 \mathrm{~dB}$ points) used for determining $Q_{0}$, and $Q$, respectively, as seen in Figure 4 . These parameters cin be printed to the CRT or an external printer for further use. If there are more data files remaining, the process is repeated; if not, arrays of $\mathrm{Q}, \mathrm{Q}_{0}, \mathrm{f}_{0}$, and $\mathrm{k}$ versus temperature are stored to a floppy disk. The user is then given the option of plotting any of the stored parameters versus temperature or terminating the program. If the user wants to plot any of the stored quantities, then the program 'DATAPLOT' can be called to do so.

Figure 5 is a flowchart of 'DATAPLOT'. The user enters which of the parameters stored by 'QANALYSIS' is to be plotted (all are plotted as a function of temperature), the graph's title and legend, and whether the data is to be plotted to the CRT or to a plotter. Once the program has verified with the user that the information entered is correct, it loads the data from the chosen file and reads it. It determines the maxima and minima of the data set, then chooses a graphing range which will center the data on the graph, as well as making the graph easy to read. Finally, it plots the data and labels the graph with a title. legend. and any other user-specitied comments.

Figure 6 shows an actual plot from 'DATAPLOT' of $Q$, for both a linear and ring resonator of $\mathrm{YBCO}$ on $\mathrm{L}_{\mathrm{aAlO}}$. Figure 7 shows plots of experimental data for a $\mathrm{YBCO}$ CBCPW resonator on $\mathrm{R}-\mathrm{Al}_{2} \mathrm{O}_{x}$. These plots give an idea of the format and scope of the plots produced by 'DATAPLOT'. The reproducibility of these data was determined by performing multiple measurement cycles. The reproducibility of $Q_{2}$ was found to be within two percent at $77 \mathrm{~K}$, and within 1.5 percent at $20 \mathrm{~K}$. The reproducibility from run-to-run for

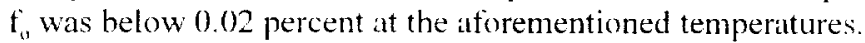

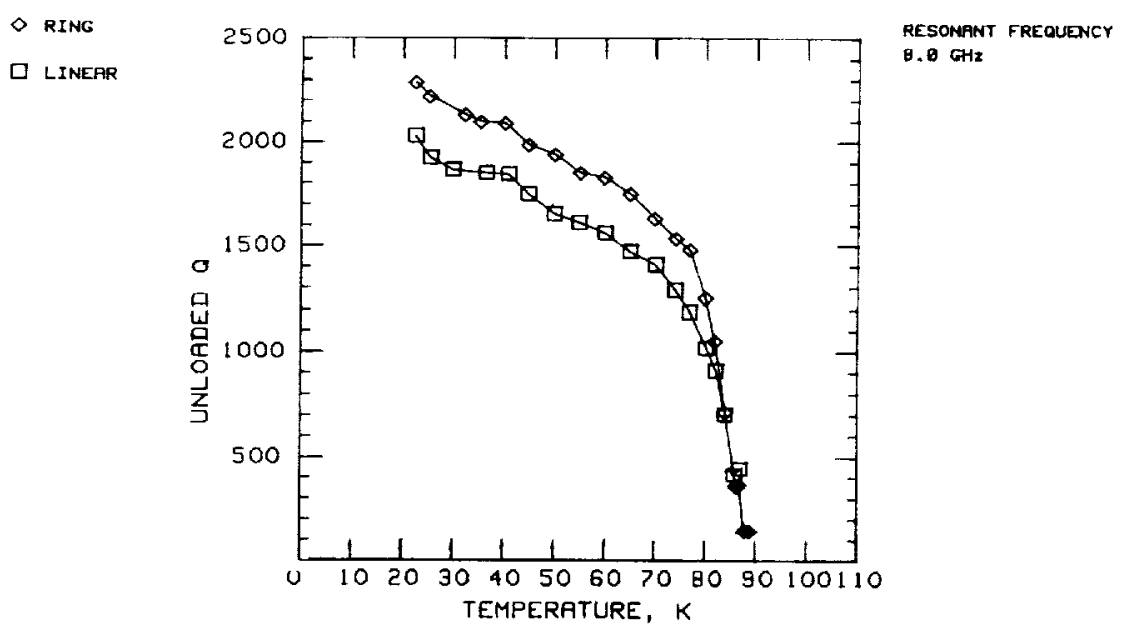

Figure 6. Sample data plot for the unloaded $\mathrm{Q}$ corresponding to $\mathrm{YBCO}$ lincar and ring resonators 

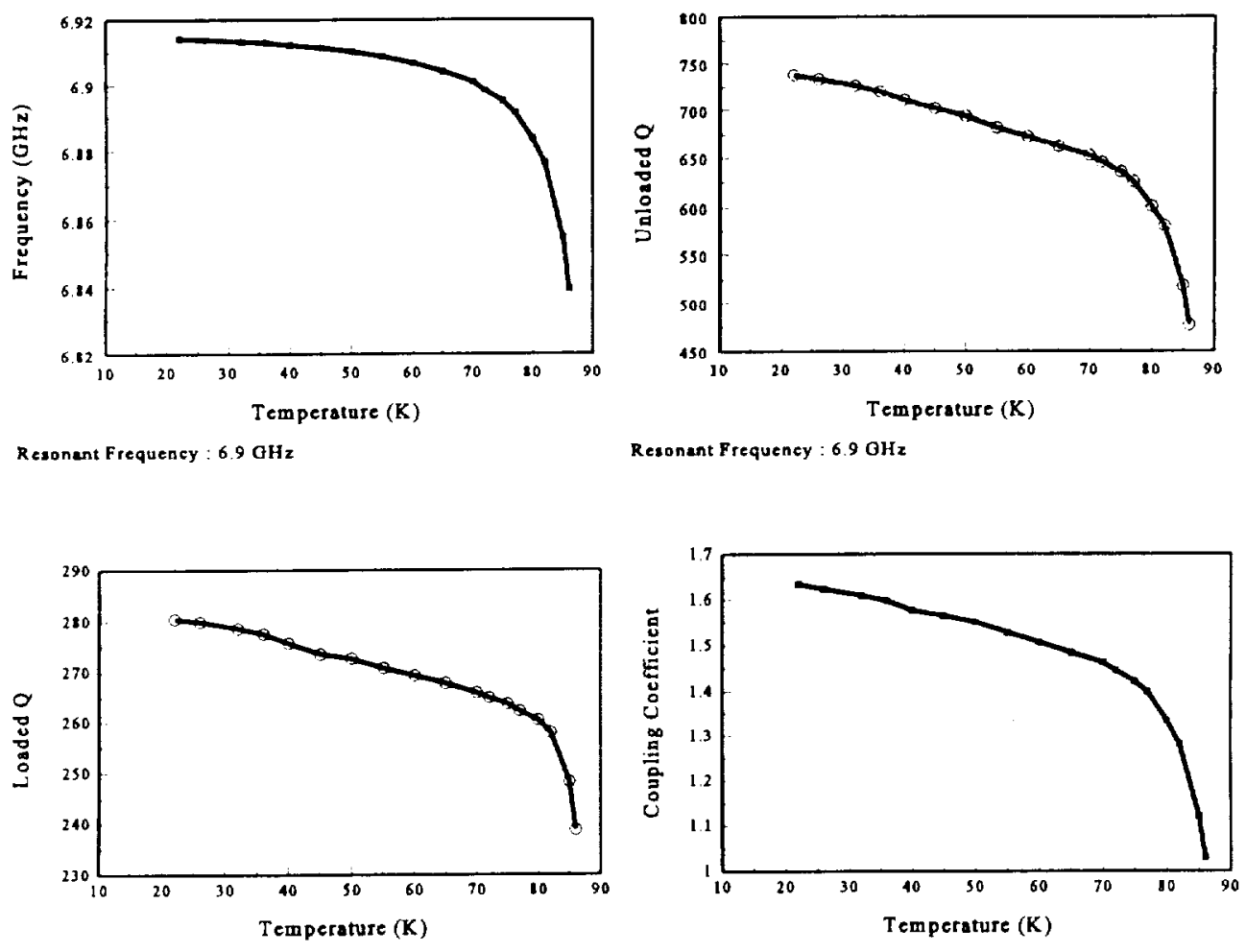

Resonent Prequency : $6.9 \mathrm{GHz}$

Resonant Frequency : $6.9 \mathrm{OHz}$

Figure 7. Plots of experimental data for a $\mathrm{YBCO}$ conductor backed coplanar waveguide resonator (CBCPW).

\section{CONCLUSIONS}

In the course of this work, automated data analysis programs which increase efficiency during the characterization of superconducting microwave resonators were developed. These programs significantly reduce the time commitment of the user in the analysis process, and provide quick access to the results of the cryogenic characterization of HTS-based resonators. Although these programs were originally developed for the study of one port (i.e., reflection mode) HTS-based resonators, they could also be used for the cryogenic characterization of two port (i.e.. reflection/transmission) resonators.

\section{ACKNOWLEDGMENTS}

The authors wish to thank Ms. Donna Bohman for patterning the HTS circuits used in this study. The authors are grateful to the referees for their constructive comments and suggestions.

\section{REFERENCES}

1. F.A. Miranda, K.B. Bhasin. K.S. Kong. T. Itoh. and M.A. Stan, Conductor backed coplanar waveguide resonators of $\mathrm{YBa}_{2} \mathrm{Cu}_{3} \mathrm{O}_{7-8}$ on $\mathrm{LaAlO}_{3}$, IEEE Microwave and Guided Wave Letters 2:287 (1992). 
2. S.H. Talisa et al., Low- and high-temperature superconducting microwave filters, IEEE Trans. Microwave The. Tech. 39:1448 (1991).

3. F.A. Miranda, Microwave properties of $\mathrm{Tl}-\mathrm{Ba}-\mathrm{Ca}-\mathrm{Cu}-\mathrm{O}$ thin films, Supercond Sci. Technol. 6:605 (1993).

4. M. Zayas, Automated measurement system for the cryogenic characterization of planar microwave components, Apogee-Proceedings of the Third Annual NASA/OAI Summer Intern Research Symposium (1993).

5. T. Edwards, "Foundations for Microstrip Circuit Design," Wiley and Sons, New York (1992).

6. R.R. Romanofsky. Analytical and experimental procedures for determining propagation characteristics of millimeter-wave gallium arsenide microstrip lines, NASA TP-2899(1989).

7. C.M. Chorey, K.S. Kong. K. B. Bhasin, J. D. Warner. and T. Itoh. YBCO ring resona tors at millimeter-wave frequencies, IEEE Trans. Microwave The. Tech. 39:1480 (1991). 